\title{
Assisting 3D Indoor Positioning for Robot Navigation
}

\author{
Ban Isam Rashid Albayati $\mathbb{D}^{\mathrm{D}}$, Serkan Kurt \\ Department of Electronics and Communication Engineering, Yıldız Technic University, İstanbul, Turkey
}

Cite this article as: Albayati BIR, Kurt S. Assisting 3D Indoor Positioning for Robot Navigation. Electrica, 2019; 19(1): 85-90.

\section{ABSTRACT}

With the increasing employment of mobile robots to achieve different tasks in various applications, the need for localization and body position for these robots is increasing rapidly. Many techniques are proposed to calculate the precise coordinates of a robot based on the distances measured between the robot and a set of reference points. Also, internal sensors, such as accelerometers and gyroscopes, are used to detect the body position and the direction of the robot. However, the effect of obstacles in an indoor environment and sensor drifts still limit the applicability of such systems. Thus, in this study, a novel technique that uses one or more robots to compensate for the missing stationary points is proposed. The robots in the proposed technique collaborate to improve the positioning accuracy, by providing reference points to each other. Per each movement execution of one robot, the remaining robots remain stationary, to provide the required reference points. When the robot finishes the movement execution, its position is updated based on the signals collected from the other robots, in addition to the position calculated by the onboard sensors. Then, another robot is selected to execute its movement.. The results show that the proposed method has been able to improve the positioning accuracy, by increasing the number of collaborating robots, when the median function if used to select the coordinates of the robot, among the candidate positions.

Keywords: Indoor positioning, majority vote, robots

\section{Corresponding Author:}

Ban Isam Rashid Albayati

E-mail:

albayati.ban90@gmail.com

Received: 25.10 .2018

Accepted: 12.01 .2019

(C) Copyright 2019 by Electrica

Available online at

http://electrica.istanbul.edu.tr

DOI: $10.26650 /$ electrica.2019.18038

\section{Introduction}

In recent years, the need for indoor positioning is rising rapidly, given the growing use of robots in accomplishing different tasks. In general, global positioning system (GPS) is used in most tasks that require positioning objects outdoors. This system uses radio waves transmitted from specialized GPS satellites orbiting around the earth. Even in outdoor positioning, the accuracy of the position computed by the GPS can vary from a few meters to tens of meters, which is not accurate enough with respect to indoor scales. Moreover, the radio waves transmitted from the GPS satellites are difficult to receive indoors, considering the obstacles they need to pass through in order to reach the receiver [1]. Thus, several studies are proposed recently to provide alternative systems for indoor positioning.

Similar to the methodology used in GPS, indoor positioning uses known coordinates as reference points to calculate the new coordinates for the object being positioned. This positioning relies on computing the distances between the given object and each of the reference points in range of that object. The new coordinates are calculated by finding all the coordinates that satisfy the measured distances from these points. Most of the earlier studies emphasize on developing techniques that improve the accuracy of the distance measured from the reference points to improve the accuracy of the calculated coordinates. Obstacles between the object and the reference points, from which the distance is being calculated, is one of the most crucial factors that affect the accuracy of the measured distance, hence, the accuracy of the calculated position [2-4].

In this study, a method is proposed to allow positioning robots that have lost communication with one or more of the stationary reference points. The remainder of this study is organized as follows: Section Two reviews previous studies related to this one; Section Three describes the proposed methodology and the computations behind it; Section Four summarizes the 
experiments conducted to evaluate the performance of the proposed method, and the results gathered from these experiments; Section Five shows the conclusions acquired from this study.

\section{Related Work}

An indoor positioning technique is proposed by Zhang et al. [5] that uses visible light to send on-off keying (OOK) messages to the receivers from light-emitting diode (LED) bulbs. Each bulb sends an identifying message that can be used to retrieve the position of that bulb in a specific time slot, to avoid receiving more than one message by the receiver. By measuring the intensity of the light at the receiving end and the angle that the light is projected on the sensor, the linear least square estimation method is used to calculate the position of the object. The results of this method show that a positioning accuracy of up to $5.9 \mathrm{~cm}$ can be achieved, using such a technique in an environment with indirect sunlight and proper installation of the LED bulbs. However, the visible light is highly affected by obstacles that may interrupt the line of sight between the bulb and the receiver and prevent the receiver from considering that reference point, or by receiving a reflection of the light that results in a wrongfully calculated distance.

The method proposed by Wang et al. [6] uses WiFi signals transmitted from access points (AP) located in known positions to calculate the position of an indoor object. The distance between the object and each AP is calculated by measuring the power of the received WiFi signal. Then, a $k$-nearest neighbor ( $k$-NN) classifier is used to integrate pseudo-odometry (P-O) with the WiFi data to eliminate the effect of obstacles, using measurements collected using pedestrian dead-rocking simulation. The results of the experiments show that a one-meter accuracy can be achieved using this approach. However, the use of the $k$-NN classifier to reduce the effect of the obstacles in the building imposes the need of collecting new simulation data for that classifier, whenever the location of the obstacles in the building are changed.

Lin et al. [7] propose an indoor positioning system that uses Bluetooth low-energy (BLE) device to transmit beacons that include identification information about the device transmitting those beacons. It is possible to position the object based on the positions of the BLE devices in range with the object by identifying that device and the distance between it and the receiving object and by measuring the received signal strength (RSS). Instead of calculating the exact coordinates of the object, the proposed system partitions each region into four sub-regions, where the position of the object is described by the sub-region that it is predicted to be in. This system is proposed to increase the efficiency of the services provided in an emergency room, which illustrates the importance of some indoor positioning applications. As the BLE devices transmit a beacon every second, the position is updated once every five seconds, in which five beacons are received in order to reduce the effect of the noise. The results show that $97.22 \%$ of the predicted positions in this technique are correct. However, as the position of the object is described using the sub-region that it is located in, no coordinates are calculated for the object to measure the accuracy of that positioning.

In addition to the coordinates of the robot, it is also important to calculate the angle and the direction that the robot is heading, in order to calibrate the gyroscope and accelerometer on the robot for short-term navigation. By calibrating these sensors, the robot gains the ability to navigate without the need of stationary points. However, such navigation suffers from drifting, in which the error caused by noise signals collected from these sensors are accumulated [8]. Different techniques are proposed to minimize such drifting, where the use of Kalman filter has shown better results by eliminating the noise from the signals collected from the sensors [9]. However, in the case of multiple robots existing in one domain, the collaboration between these robots can improve the position computations, where each robot uses the positions of other robots, calculated based on their gyroscopes and accelerometers.

\section{Method}

To calculate the 3-dimensional coordinates of an object using reference points, the distance between that object and a minimum of four reference points, alongside with their coordinates, is required. By measuring the distance between the object and a single reference point, the object may have coordinates that position it anywhere in the circumference of a sphere centered at each reference point, with a radius equal to the measured distance. Using another reference point, the object may have coordinates that position it anywhere in the circle that results from intersecting the spheres centered at these reference points. Using the third reference point, the coordinates are limited to only two possible locations, which are at intersects of the circle computed from the first two points with the sphere of the third. To obtain the coordinates of the object between these two candidate points, a fourth sphere centered at the fourth reference point is used, where the point that belongs to the circumference of that sphere is selected among those two candidates. The equation of the sphere centered at the reference point with coordinates $\left(x_{1}, y_{1}, z_{1}\right)$ and radius of $r_{1}$ is shown as

$\left(x-x_{1}\right)^{2}+\left(y-y_{1}\right)^{2}+\left(z-z_{1}\right)^{2}=r_{1}^{2}$

Using (1) and the equations of spheres from other reference points, the following can be concluded.

$$
\begin{aligned}
& x^{2}-2 x x_{1}+x_{1}^{2}+y^{2}-2 y y_{1}+y_{1}^{2}+z^{2}-2 z z_{1}+z_{1}^{2} \\
& =r_{1}^{2} \\
& x^{2}-2 x x_{2}+x_{2}^{2}+y^{2}-2 y y_{2}+y_{2}^{2}+z^{2}-2 z z_{2}+z_{2}^{2} \\
& =r_{2}^{2}
\end{aligned}
$$




$$
\begin{aligned}
& x^{2}-2 x x_{3}+x_{3}^{2}+y^{2}-2 y y_{3}+y_{3}^{2}+z^{2}-2 z z_{3}+z_{3}^{2} \\
& =r_{3}^{2} \\
& x^{2}-2 x x_{4}+x_{4}^{2}+y^{2}-2 y y_{4}+y_{4}^{2}+z^{2}-2 z z_{4}+z_{4}^{2} \\
& =r_{4}^{2}
\end{aligned}
$$

To eliminate the squares of the variables $x^{2}, y^{2}$ and $z^{2},(5)$ is subtracted from (2), (3), and (4) which illustrates the use of the fourth reference point to select one of two candidate points calculated using

$$
\begin{aligned}
& x\left(2 x_{1}-2 x_{4}\right)+y\left(2 y_{1}-2 y_{4}\right)+z\left(2 z_{1}-2 z_{4}\right)=r_{4}^{2}-r_{1}^{2}+x_{1}^{2}-x_{4}^{2}+y_{1}^{2}-y_{4}^{2}+z_{1}^{2}-z_{4}^{2} \\
& x\left(2 x_{2}-2 x_{4}\right)+y\left(2 y_{2}-2 y_{4}\right)+z\left(2 z_{2}-2 z_{4}\right)=r_{4}^{2}-r_{2}^{2}+x_{2}^{2}-x_{4}^{2}+y_{2}^{2}-y_{4}^{2}+z_{2}^{2}-z_{4}^{2} \\
& x\left(2 x_{3}-2 x_{4}\right)+y\left(2 y_{3}-2 y_{4}\right)+z\left(2 z_{3}-2 z_{4}\right)=r_{4}^{2}-r_{3}^{2}+x_{3}^{2}-x_{4}^{2}+y_{3}^{2}-y_{4}^{2}+z_{3}^{2}-z_{4}^{2} \\
& {\left[\begin{array}{lll}
\left(2 x_{1}-2 x_{4}\right) & \left(2 y_{1}-2 y_{4}\right) & \left(2 z_{1}-2 z_{4}\right) \\
\left(2 x_{2}-2 x_{4}\right) & \left(2 y_{2}-2 y_{4}\right) & \left(2 z_{2}-2 z_{4}\right) \\
\left(2 x_{3}-2 x_{4}\right) & \left(2 y_{3}-2 y_{4}\right) & \left(2 z_{3}-2 z_{4}\right)
\end{array}\right]^{-1} \times\left[\begin{array}{l}
\left(r_{4}^{2}-r_{1}^{2}+x_{1}^{2}-x_{4}^{2}+y_{1}^{2}-y_{4}^{2}+z_{1}^{2}-z_{4}^{2}\right) \\
\left(r_{4}^{2}-r_{2}^{2}+x_{2}^{2}-x_{4}^{2}+y_{2}^{2}-y_{4}^{2}+z_{2}^{2}-z_{4}^{2}\right) \\
\left(r_{4}^{2}-r_{3}^{2}+x_{3}^{2}-x_{4}^{2}+y_{3}^{2}-y_{4}^{2}+z_{3}^{2}-z_{4}^{2}\right)
\end{array}\right]=\left[\begin{array}{l}
x \\
y \\
z
\end{array}\right]}
\end{aligned}
$$

the other reference points, i.e., the square root of these variables, where $\sqrt{x^{2}}= \pm x$, for example. This subtraction results the equations (6), (7) and (8). The values of $x, y$, and $z$, which represent the coordinates of the object, can then be calculated from these equations using the matrices operations shown in (9). Regardless of which equation is subtracted from the other equations, the described procedure always results in the same coordinates. However, the use of a fifth reference point can result in four different combinations of equations that can be used to calculate four coordinates for the object. Moreover, when a new reference point is added to the existing set of points, four more combinations can be added to the calculations of the position, which results in a more accurate computation.

Moreover, as each robot has a gyroscope and an accelerometer that can be used to calculate the distance traveled by the robot, with respect to the last known position, the last position of the robot can also be considered as a reference point, while the distance from that point is calculated using the onboard sensors. However, the effect of the drifting can occur in these sensors, resulting in an inaccurate positioning, unless other reference points are used to improve the positioning accuracy. Thus, in addition to the use of the onboard sensors to calculate the distance traveled by the robot, it is possible to use only three reference points to position the robot but a perfect positioning accuracy cannot be guaranteed, when considering the drifting effect.

In the proposed method, the robot is expected to have access to four reference points under normal operation. However, in certain conditions, the robot may end up in certain positions where the stationary reference points are no longer accessible. At such positions, one or more robots are sent to assist the stuck robot in recalculating its position. By making use of the collaboration among these devices, it is possible to improve the accuracy of the positioning of each of these robots.
When one robot moves, the remaining robots are required to stay in stationary, i.e., they do not change their positions. As soon as the requested movement command is completed by the first robot, according to the measurements collected from the gyroscope and the accelerometer, this robot becomes stationary as well. Then, the coordinates of its new position are calculated based on the measurements collected from its sensors as well as the distances collected from the other robots and stationary reference points, if they exist. The calculated position is then used by this robot instead of the older coordinates, calculated based on the gyroscope and the accelerometer. At this instance, a command is sent to another robot to execute the required movement.

As the ratio of robots used to assist the positioning process increases, the effect of drifting that occurs in the onboard sensors of the calculated coordinates increase, with respect to the stationary reference points. However, as the total number of reference points used in the coordinate's calculations, including the last position and the distance measured using the onboard sensors, exceeds four reference points, multiple candidate positions can be calculated. Selecting certain coordinates from the candidates list can be achieved by averaging these candidates, or by selecting the median value.

\section{Experiments}

To evaluate the performance of the proposed method, a model is created using MATLAB development environment. A mobile robot is simulated to travel from a position where the positioning process relies on four stationary reference points to a position where no stationary reference points are accessible. Thus, the robot starts losing the signals incoming from these stationary points, one by one.

As soon as the first signal is lost, the robot starts to use its onboard accelerometer and gyroscope to calculate the distance traveled from one position to another. However, another robot is sent to that robot to assist in the positioning process, and by performing various random motions while depending on its onboard accelerometer and gyroscope, the stuck robot can calculate its new location. When another signal is lost, another assisting robot is sent, until the entire positioning process becomes based on the collaborating robots, while the stationary reference points have no role in the positioning process. When a robot is moving, the positions of the remaining robots are fixed. A maximum of $1 \%$ of the traveled distance is simulated as an error in the calculated distance between the current and previous positions, to cover the drifting effect of the onboard sensors.

When more than one candidate set or coordinates are available, the position of the robot is selected using the average and median functions. By the end of the trip, the mean error in the actual and calculated positions is calculated for the positions of both functions. The robot is set to travel to the destination coordinates in five steps. Per each step the position 
of the robot is fixed and the remaining robots are moved, one by one. Per each position where a robot requires assistance, three robots are used to evaluate the positioning accuracy. The first robot requires the use of onboard sensors to satisfy the minimum of four reference positions. The second robot allows the computations using four reference points, including the onboard sensors, while the third robot acts as an extra in order to achieve a more precise computation. The mean error per each experiment, for the average and median functions, is shown in Table 1.The results show that the use of the median function has been able to achieve more accurate positioning. In addition to the known immunity of the median function against noise values, the use of the average function to select the position of the robot from the candidate coordinates has reduced the positioning accuracy. This reduction is a result of the accumulated drifting that occurs in the accelerometer and gyroscope sensors. Moreover, the results show that the positioning accuracy based on the median function increases when the number of assisting robots are increased, but it is reduced when the average function is used instead.

Table 1. Mean error measured for the different steps of the robot

\begin{tabular}{cccc}
\hline \multicolumn{2}{c}{ Reference Points } & \multicolumn{2}{c}{ Mean Error (\%) } \\
\hline Stationary & Robots & Average & Median \\
\hline 4 & 0 & 0 & 0 \\
\hline 3 & 0 & 0.17 & 0.17 \\
\hline 3 & 1 & 0.23 & 0.07 \\
\hline 3 & 2 & 0.21 & 0 \\
\hline 2 & 1 & 0.34 & 0.34 \\
\hline 2 & 2 & 0.34 & 0.13 \\
\hline 2 & 3 & 0.29 & 0.08 \\
\hline 1 & 2 & 0.42 & 0.42 \\
\hline 1 & 3 & 0.56 & 0.38 \\
\hline 1 & 4 & 0.58 & 0.31 \\
\hline 0 & 3 & 0.77 & 0.76 \\
\hline 0 & 4 & 0.78 & 0.59 \\
\hline 0 & 5 & 0.83 & 0.46 \\
\hline
\end{tabular}

\section{Conclusion}

The focus on indoor positioning is increasing recently, based on the rapid growth of using automated robots in different tasks. Many techniques are proposed to provide these robots with precise coordinates by measuring the distance between each robot and a set of stationary reference points, located in known positions in the environment, or based on the onboard accelerometer and gyroscope sensors. However, obstacles in the environment are one of the primary challenges that are faced by indoor positioning techniques, whereas an obstacle may block the communication between the robot and the reference points or affect it sufficiently to result in wrong distance measurements, while drifting is the main problem that faces the use of onboard sensors to calculate the coordinates of the robot. This drifting occurs due to the accumulating effect of noise signals collected by the sensors.

Moreover, robots that end up in blind spots, where the number of stationary reference points that are within the range of the robot in that environment is not sufficient to accurately calculate the coordinates, can no longer calculate their positions. Relying on the onboard sensors accumulates to significant differences between the calculated and actual coordinates. Thus, a contemporary technique is proposed in this study to enable robots of being positioned with the assistance of a designated one or more robot that transmit signals similar to those transmitted by the stationary reference points and uses their positions to assist robots that have lost communication with the stationary reference points calculating their positions.

The results of the simulated model show that the collaboration among the robots to improve their positioning accuracy has significantly improved when the median function is used to select the coordinates of a robot from the candidate coordinates. The existence of more than three external reference points, other than the use of onboard sensors and previous positions, can produce multiple candidate positions. The average and median functions are used to calculate the coordinates of the robot, based on those candidates. The average function has shown high sensitivity to the accumulated error of the onboard sensors, which is accumulated further when the robot executes other steps. However, the median function has been able to eliminate such accumulations by improving the positioning of each step.

Increasing the number of robots that collaborate in the positioning process has improved the positioning accuracy when the median function is used in the computations, while this accuracy is reduced when the average function is used. This behavior in the average-based computations is caused by the accumulating error caused by the drifting of all the sensors on the robots, where each robot executes its own movement while the others are in stationery. In the median-based computations, the error is limited to the drifting that occurs in the robot that have the selected coordinates.

As a future work, the ability of sending one or more robot to a position where the stationary reference points are accessible may be evaluated. Such an approach can be used to reset any accumulated drifting that occurs in the computations in order to improve the positioning accuracy. 
Peer-review: Externally peer-reviewed.

Conflict of Interest: The authors have no conflicts of interest to declare.

Financial Disclosure: The authors declared that this study has received no financial support.

\section{References}

1. R. Momose, T. Nitta, M. Yanagisawa, N. Togawa, "An accurate indoor positioning algorithm using particle filter based on the proximity of bluetooth beacons", in Consumer Electronics (GCCE), 2017 IEEE $6^{\text {th }}$ Global Conference, pp. 1-5, 2017. [CrossRef]

2. S. He, S. H. G. Chan, "Wi-Fi fingerprint-based indoor positioning: Recent advances and comparisons", IEEE Communications Surveys \& Tutorials, vol. 18, pp. 466-490, 2016. [CrossRef]

3. A. Alarifi, A. Al-Salman, M. Alsaleh, A. Alnafessah, S. Al-Hadhrami, M. A. Al-Ammar, eAl-Khalifa HS, "Ultra wideband indoor positioning technologies: Analysis and recent advances", Sensors, vol. 16, p. 707, 2016. [CrossRef]
4. P. Nazemzadeh, F. Moro, D. Fontanelli, D. Macii, L. Palopoli, "Indoor positioning of a robotic walking assistant for large public environments", IEEE Transactions on Instrumentation and Measurement, vol. 64, pp. 2965-2976, 2015. [CrossRef]

5. W. Zhang, M. S. Chowdhury, M. Kavehrad, "Asynchronous indoor positioning system based on visible light communications", Optical Engineering, vol. 53, p. 045105, 2014. [CrossRef]

6. J.Wang, A. Hu, C. Liu, X. Li, "A floor-map-aided WiFi/pseudo-odometry integration algorithm for an indoor positioning system", Sensors, vol. 15, pp. 7096-7124, 2015. [CrossRef]

7. X.-Y. Lin, T.-W. Ho, C.-C. Fang, Z.-S. Yen, B.-J. Yang, F. Lai, "A mobile indoor positioning system based on iBeacon technology", in Engineering in Medicine and Biology Society (EMBC), 2015 37th Annual International Conference of the IEEE, pp. 4970-4973, 2015.

8. L. P. N. Sekar, A. Santos, O. Beltramello, "IMU Drift Reduction for Augmented Reality Applications", in International Conference on Augmented and Virtual Reality, pp. 188-196, 2015.

9. M. Narasimhappa, A. D. Mahindrakar, V. C. Guizilini, M. H. Terra, S. L. Sabat, "An improved Sage Husa adaptive robust Kalman Filter for de-noising the MEMS IMU drift signal", in Indian Control Conference (ICC). 2018, pp. 229-234, 2018. [CrossRef] 

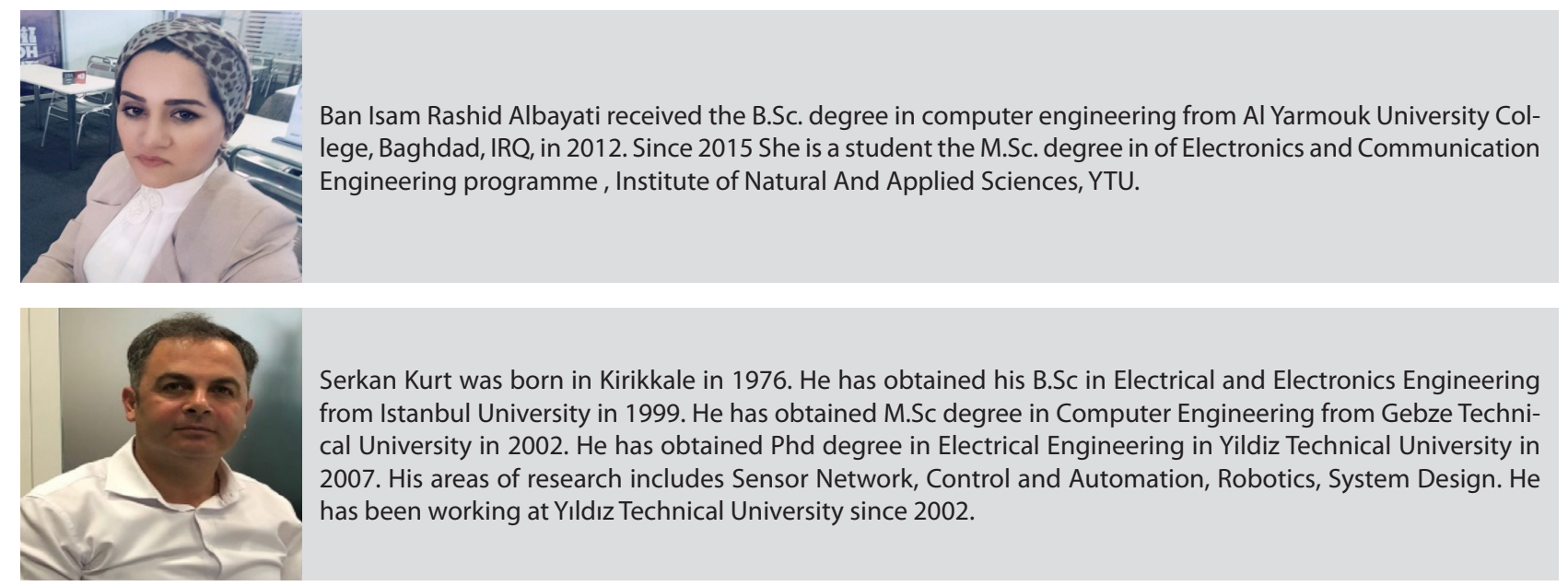